of these 3 buffalo sera did not parallel those of any known cattle reagent. Absorptions with different cattle cells suggested that each serum contained a multiplicity of antibodies directed against unknown factors.

The reactivity of $F, V$ and $Z$ reagents with bison cells ${ }^{2-4}$ and their failure to react with water buffalo cells suggest that serological relationship between water buffaloes and cattle is less close than between bison and cattle. Our results demonstrate that an antigenic system similar to $J$ of cattle occurs in water buffaloes. This is another example of the ubiquity of the $J$ blood-group substance. Whether or not the $J$-like substance of water buffaloes is identical with the $J$ substance of cattle must await more data.

This work was supported in part by the Research Committee of the Graduate School from funds supplied by Wisconsin Alumni Research Foundation, and by a research grant $(E-1643)$ from the U.S. National Institutes of Health.

We thank the Director, Indian Veterinary Research Institute, Izatnagar (U.P.), India, for use of animals and laboratory facilities.

Department of Genetics,

S. P. Datta

University of Wisconsin, Madison.

${ }^{1}$ Stormont, C., Ann. N.Y. Acad. Sci., 97, 251 (1962).

${ }^{2}$ Miller, W. J., and Stone, W. H., Genetics, 40, 586 (1955).

${ }^{3}$ Owen, R. D., Stormont, C., and Irwin, M. R., Evolution, 12, 102 (1958).

4 Stormont, C., Miller, W. J., and Suzuki, Y., Evolution, 15, 196 (1961).

${ }^{5}$ Gasparski, J., and Dubiski, S., Ann. N.Y. Acad. Sci., 97, 285 (1962).

- Eyquem, A., Podliachouk, L., and Millot, P., Ann. N.Y. Acad. Sci., 97, $320(1962)$

'Singh, B., Ind. J. Vet. Sci. Anim. Husb., 12, 12 (1942).

${ }^{8}$ Stone, W. H., and Irwin, M. R., J. Immunol., 73, 397 (1954).

'Conneally, P. M., Patel, J. R., Morton, N. F., and Stone, W. H., Genetics, 47, 797 (1962).

\section{Tests for Linkage of the Genes controlling Cattle Transferrins and Blood Groups}

There are 11 genetically independent blood-group systems (loci) in cattle ${ }^{1}$ which provide markers on perhaps a third of their chromosomes. Attempts to discover linkage between these blood-group genes and those controlling production traits have generally been unsuccessful ${ }^{2}$. Recent discoveries of various inherited biochemical polymorphisms such as transferrins, hæmoglobins, $\beta$-lactoglobulins, $\alpha$-lactoalbumins, etc. ${ }^{3}$, now provide additional markers for linkage investigations. This report presents the results of tests for linkage between the transferrin locus and seven of the blood-group loci.

Transferrin and blood-group data used in this analysis were obtained from Holstein-Friesian herds at Pabst Farms, Oconomowoc, Wisconsin; Emmons Blain, jun., Experimental Farm, Lake Mills, Wisconsin; and the Dairy Cattle Research Center, University of Wisconsin, Madison. Transferrin types were determined by vertical starch-gel electrophoresis using borate buffer ${ }^{4}$. The blood types were determined by the hæmolytic test ${ }^{5}$ routinely used in our Immunogenetics Laboratory.

Linkage tests were performed by the sequential probability ratio test ${ }^{6}$. The heterozygote is distinguishable from the homozygote for transferrin type and for the $F$ and $Z$ blood-groups, but the genotypes of the other five blood-group loci had to be ascertained from the pedigree information. Individuals with doubtful genotypes were not used. Only families of sires heterozygous for both the transferrin (main locus) and blood-group loci (test loci) were used. The double and single backcrosses were the only informative matings. Lod scores were determined from the $Z_{1}$ tables computed by Morton ${ }^{6}$.

Table 1 gives the accumulated lod scores from tests for linkage between the transferrin locus and each of the seven blood-group loci. The significance of the accumulated $Z$ scores was tested by comparing $\Sigma Z$ with $\log A$ and $\log$ $B$. As recommended by Morton ${ }^{6}, \log B=-2$ and $\log$
Table 1. Results of Sequentiaf Probabluity Ratio Tests for Linkagh BETWEEN TRANSFERRIN LOOUS AND BLOOD-GROUP LOCI

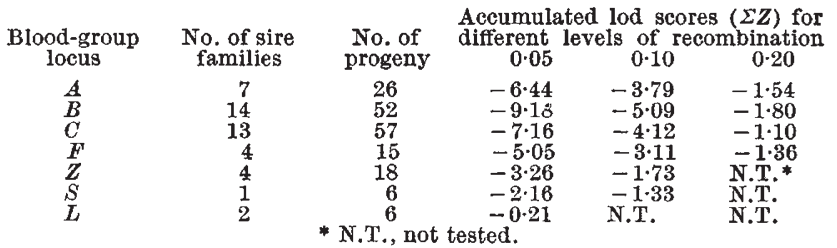

$A=3$ were taken as appropriate choices. Linkage of the order of 10 per cent recombination was excluded for bloodgroup loci $A, B, C$ and $F$. For the $Z$ and $S$ loci, linkage at the level of 5 per cent recombination could be excluded. The data were not sufficient to exclude linkage with the $L$ locus even at the level of 5 per cent recombination. Thus, there is yet no clear-cut linkage detectable between the blood-group loci and any other locus in cattle.

This work was supported in part by the Research Committee of the Graduate School from funds supplied by Wisconsin Alumni Research Foundation and by a research grant ( $E$-3204) from the U.S. National Institutes of Health.

We thank Dr. N. E. Morton for advice and assistance and the many persons who provided the blood samples.

S. P. Datta.

Department of Genetics,

W. H. STONE

University of Wisconsin, Madison.

${ }^{1}$ Morton, N. E., Stone, W. H., and Irwin, M. R., Genetics, 41, 655 (1956).

${ }^{2}$ RendeI, J., Z. Tierz. Ziicht., 75, 97 (1961).

${ }^{3}$ Ogden, A. L., Animal Breed. Abst., 29, 127 (1961).

4 Smithies, O., Adv. Protein Chem., 14, 65 (1959).

${ }^{5}$ Stormont, C., Proc. Tenth Intern. Cong. Genetics, 1 (1959).

- Morton, N. E., Amer. J. Hum. Genetics, 7, 277 (1955).

\section{Diploid-like Pairing in a Synthetic Decaploid}

IN the course of investigations on species relationships within the genus Avena, a number of pentaploid hybrids derived from the cross Avena abyssinica Hochst. $(2 n=28)$ $\times A$. sativa L. (Black Mesdag) $(2 n=42)$ were successfully treated with colchicine using the capping technique ${ }^{1}$. Thirty-six seeds were obtained from the doubled sectors of the treated pentaploid. Chromosome pairing was examined in four pentaploid hybrids and five decaploid plants. Twenty cells per plant were analysed and a short account of chromosome pairing at $M_{1}$ in both pentaploid and decaploid forms is given in Tables 1 and 2.

Table 1. Chromosome Pairing in the Pentaploid Hybrids

\begin{tabular}{ccccc} 
& \multicolumn{4}{c}{ Mean No. of configurations per cell } \\
Hybrid No. & I & II & III & IV \\
$4411 \mathrm{Cn}$ & $21 \cdot 35$ & $4 \cdot 71$ & $1 \cdot 10$ & $0 \cdot 21$ \\
$\mathbf{4 4 1 2} \mathrm{Cn}$ & 22.25 & $4 \cdot 14$ & $1 \cdot 00$ & $0 \cdot 25$ \\
$\mathbf{4 4 1 3} \mathrm{Cn}$ & 18.03 & $5 \cdot 41$ & $1 \cdot 86$ & $0 \cdot 29$ \\
$4415 \mathrm{Cn}$ & $19 \cdot 22$ & $4 \cdot 86$ & $1 \cdot 77$ & $0 \cdot 18$
\end{tabular}

On the basis of their investigations of chromosome morphology ${ }^{2}$ and genome homology ${ }^{3}$ in the Avenae, Rajhathy and Morrison concluded that there is only one common genome in the tetraploid and hexaploid species. They designated the genomic constitution of the hexaploid species as AACCDD and the tetraploids as AABB, and accordingly the expected theoretical pairing in the pentaploid would be 21 univalents and 7 bivalents. Some pairing between chromosomes of the $A$ and $B$ genome has been previously reported by Nishiyama and the presence of multivalents in the pentaploid hybrids may be an indication of similar pairing.

According to the foregoing designation of the genomic relationships in the Avenae ${ }^{2,3}$ the decaploid would be AAAABBCCDD with an expected chromosome pairing of up to a maximum of 7 quadrivalents and 21 bivalents. Lesik $^{5}$ reported 35 bivalents for a similar decaploid 